\title{
Characterization of the Aspergillus fumigatus detoxification systems for reactive nitrogen intermediates and their impact on virulence
}

\author{
Katrin Lapp ${ }^{1}$, Martin Vödisch ${ }^{1}$, Kristin Kroll ${ }^{1}$, Maria Strassburger ${ }^{1}$, Olaf Kniemeyer ${ }^{1,2,3}$, \\ Thorsten Heinekamp ${ }^{1,2} *$ and Axel A. Brakhage ${ }^{1,2} *$
}

${ }^{1}$ Department of Molecular and Applied Microbiology, Leibniz Institute for Natural Product Research and Infection Biology - Hans Knöll Institute, Jena, Germany

${ }^{2}$ Department of Microbiology and Molecular Biology, Institute of Microbiology, Friedrich Schiller University Jena, Jena, Germany

${ }_{3}^{3}$ Integrated Research Treatment-Center - Center for Sepsis Control and Care, University Hospital Jena, Jena, Germany

\section{Edited by:}

Alex Andrianopoulos, University of

Melbourne, Australia

\section{Reviewed by:}

Elaine M. Bignell, The University of

Manchester, UK

Sven Krappmann,

Friedrich-Alexander-Universität

Erlangen-Nürnberg - University

Hospital Erlangen, Germany

*Correspondence:

Axel A. Brakhage and Thorsten Heinekamp, Department of Molecular and Applied Microbiology, Leibniz Institute for Natural Product Research and Infection Biology - Hans Knöll Institute, Adolf-Reichwein-Straße 23, 07745 Jena, Germany

e-mail: axel.brakhage@hki-jena.de, thorsten.heinekamp@hki-jena.de
Aspergillus fumigatus is a saprophytic mold that can cause life-threatening infections in immunocompromised patients. In the lung, inhaled conidia are confronted with immune effector cells that attack the fungus by various mechanisms such as phagocytosis, production of antimicrobial proteins or generation of reactive oxygen intermediates. Macrophages and neutrophils can also form nitric oxide (NO) and other reactive nitrogen intermediates (RNI) that potentially also contribute to killing of the fungus. However, fungi can produce several enzymes involved in RNI detoxification. Based on genome analysis of $A$. fumigatus, we identified two genes encoding flavohemoglobins, FhpA, and FhpB, which have been shown to convert NO to nitrate in other fungi, and a gene encoding $S$-nitrosoglutathione reductase GnoA reducing $S$-nitrosoglutathione to ammonium and glutathione disulphide. To elucidate the role of these enzymes in detoxification of RNI, single and double deletion mutants of FhpA, FhpB, and GnoA encoding genes were generated. The analysis of mutant strains using the NO donor DETA-NO indicated that FhpA and GnoA play the major role in defense against RNI. By generating fusions with the green fluorescence protein, we showed that both FhpA-eGFP and GnoA-eGFP were located in the cytoplasm of all $A$. fumigatus morphotypes, from conidia to hyphae, whereas FhpB-eGFP was localized in mitochondria. Because fhpA and gnoA mRNA was also detected in the lungs of infected mice, we investigated the role of these genes in fungal pathogenicity by using a murine infection model for invasive pulmonary aspergillosis. Remarkably, all mutant strains tested displayed wild-type pathogenicity, indicating that the ability to detoxify hostderived $\mathrm{RNI}$ is not essential for virulence of $A$. fumigatus in the applied mouse infection model. Consistently, no significant differences in killing of $\Delta f h p A, \Delta f h p B$, or $\Delta g n o A$ conidia by cells of the macrophage cell line MH-S were observed when compared to the wild type.

Keywords: Aspergillus fumigatus, reactive nitrogen intermediates, nitric oxide, virulence

\section{INTRODUCTION}

The filamentous mold Aspergillus fumigatus is a ubiquitous saprophyte, degrading organic matter and thereby playing a key role in recycling a variety of carbon and nitrogen sources (Tekaia and Latgé, 2005). From a medical point of view A. fumigatus is also an important pathogen of humans (Brakhage, 2005). Diseases caused by $A$. fumigatus are wide-ranging, from allergies in immunocompetent hosts to systemic infections with invasive growth in patients with a severely weakened immune system, e.g., due to therapy of hematological malignancies, after stem cell or solid organ transplantation or suffering from chronic granulomatous disease. In immunocompromised patients, the lung is the major site of infection of A. fumigatus. The most prominent immune effector cells in the lung are alveolar macrophages and neutrophil granulocytes (Bogdan et al., 2000). Macrophages phagocyte resting spores and kill them mainly through oxygen dependent mechanisms (Philippe et al., 2003). Since contact with
A. fumigatus hyphae induces an oxidative burst in neutrophil granulocytes, which is coupled to degranulation and the secretion of reactive oxygen intermediates (ROI) and also reactive nitrogen intermediates (RNI), an important role of these reactions in killing of A. fumigatus was suggested. However, deletion of the genes of two main regulators of oxidative stress response of $A$. fumigatus, the bZIP transcription factor AfYapl and the stress response regulator $A f S k n 7$, did not affect virulence of the fungus in a mouse infection model (Lamarre et al., 2007; Lessing et al., 2007; Brakhage et al., 2010). Lambou et al. (2010), however, demonstrated that ROS detoxification by superoxide dismutases contributes to resistance against killing by murine macrophages but not to virulence. Since the importance of ROI for direct killing of A. fumigatus is therefore questionable, the role of RNI on the killing of $A$. fumigatus awaits further analysis. Nitric oxide (NO) is produced by the inducible NO synthase (iNOS) of host immune effector cells. After pathogen recognition, iNOS is stimulated by 


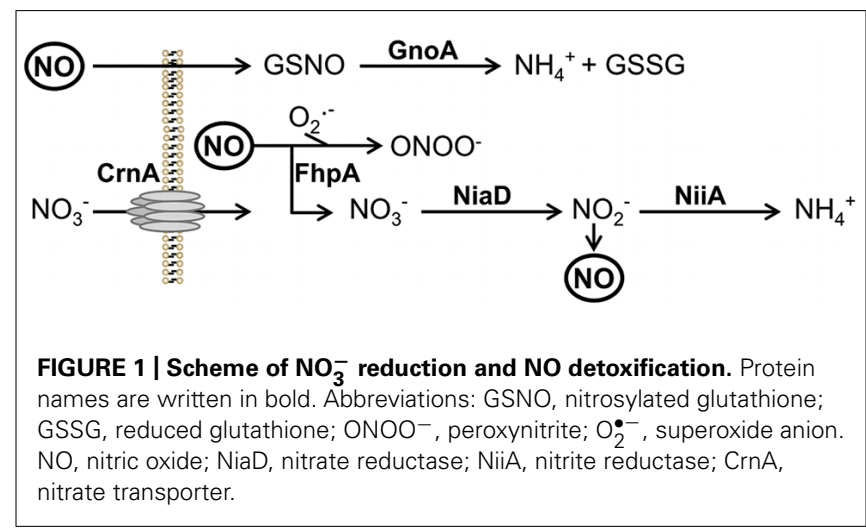

similar cytokines that are also required for induction of ROI production, e.g., IFN- $\gamma$, TNF- $\alpha$, and IL-1 (Fang, 1997). It was shown that macrophages can produce up to $14 \mathrm{mM}$ hydrogen peroxide and $57 \mu \mathrm{M}$ NO (Brown et al., 2009). It is therefore likely that both radicals are present in infected tissue and that they interact to form highly reactive intermediates such as peroxynitrite (see Figure 1).

Microorganisms have evolved several protective mechanisms to counteract nitrosative stress. Reduced glutathione plays an essential role to survive nitrosative stress. At the same time, catalases and superoxide dismutases detoxify ROI and prevent formation of peroxynitrite. During moderate oxidative stress NO radicals are detoxified via nitrite or nitrate intermediates. In the pathogenic yeast Candida albicans the flavohemoglobin YHB1 was shown to be specifically involved in detoxification of NO radicals. Expression of $y h b 1$ was induced by contact of the fungus with macrophages (Chiranand et al., 2008). A C. albicans yhb1 $\Delta / y h b 1 \Delta$ mutant displayed moderately attenuated virulence in a murine infection model of disseminated candidiasis. However, the virulence defect of the C. albicans $y h b 1 \Delta / y h b 1 \Delta$ mutant was not suppressed in mice defective for the NO synthase 2 (NOS2), indicating that not the reduced ability to detoxify RNI but another defect of the $y h b 1 \Delta / y h b 1 \Delta$ mutant is the underlying cause for attenuation in virulence (Hromatka et al., 2005).

In Cryptococcus neoformans expression of glutathione reductase and thioredoxin peroxidase was induced by nitrosative stress. A glutathione reductase mutant was hypersensitive against NO and avirulent in a mouse infection model (Missall et al., 2006). The flavohemoglobin Fhb1 of C. neoformans catalyses the conversion of NO to nitrate. During nitrosative stress NO reacts with intracellular glutathione to $S$-nitrosoglutathione which is then reduced by the enzyme $S$-nitrosoglutathione reductase (Gnol) to form again oxidized glutathione (Fernández et al., 2003). Both genes, gnol and $f h b 1$, are essential for virulence of $C$. neoformans (de Jesús-Berríos et al., 2003).

Here, we identified two flavohemoglobins, the cytosolic FhpA and the mitochondrial FhpB, and, in addition, the $S$-nitrosoglutathione reductase GnoA of $A$. fumigatus. We comprehensively analyzed the role of the respective genes fhpA (AFUA_4G03410), fhpB (AFUA_8G06080), and gnoA (AFUA_2G01040) with regard to detoxification of RNI and their impact on virulence.

\section{MATERIALS AND METHODS ETHICS STATEMENT}

Mice were cared for in accordance with the principles outlined by the European Convention for the Protection of Vertebrate Animals Used for Experimental and Other Scientific Purposes (http://conventions.coe.int/Treaty/en/Treaties/Html/123.htm). All animal experiments were in compliance with the German animal protection law and were approved by the responsible Federal State authority "Thüringer Landesamt für Lebensmittelsicherheit und Verbraucherschutz" and ethics committee "Beratende Kommission nach $\$ 15$ Abs. 1 Tierschutzgesetz” with the permit Reg.-Nr. 03-001/12.

\section{STRAINS, MEDIA AND CULTIVATION CONDITIONS}

All A. fumigatus strains used in this study are listed in Table S1. A. fumigatus strain CEA17 $\triangle a k u B$ (da Silva Ferreira et al., 2006) was used to generate mutant strains $\Delta f h p A, \Delta f h p B, \Delta g n o A$, $\Delta g n o A / \triangle f h p A, \Delta g n o A / \Delta f h p B$, and $\Delta f h p A / \Delta f h p B$. The strains were cultivated in Aspergillus minimal medium (AMM; Brakhage and Van den Brulle, 1995) containing $50 \mathrm{mM}$ glucose and $70 \mathrm{mM}$ $\mathrm{NaNO}_{3}$ as sole carbon and nitrogen source, respectively, if not otherwise stated. Alternative nitrogen sources were ammonium tartrate $(20 \mathrm{mM})$ or glutamine $(20 \mathrm{mM})$.

\section{STANDARD DNA TECHNIQUES, RNA EXTRACTION, cDNA SYNTHESIS, AND RT-PCR}

Standard techniques for manipulation of DNA, including isolation of genomic A. fumigatus DNA and Southern blot analyses, were carried out as described earlier (Wartenberg et al., 2011). For RNA isolation and Northern blot analyses the protocols previously described (Bergmann etal., 2010) were followed. RNA samples were incubated with Turbo DNA-free ${ }^{\mathrm{TM}}$ Kit (Ambion, USA) and transcribed in CDNA with the RevertAid ${ }^{\mathrm{TM}}$ Premium First Strand cDNA Synthesis Kit according to the manufacturer's protocol (Fermentas, Germany).

\section{GENERATION OF MUTANT STRAINS}

Transformation of A. fumigatus protoplasts was performed as described earlier (Weidner et al., 1998). For deletion of gnoA (AFUA_2G01040) the flanking regions were amplified from genomic $A$. fumigatus DNA with the primer pairs gnoA_5for/gnoA_ptrA_5rev and gnoA_3rev/gnoA_ptrA_3for. The pyrithiamine resistance cassette $\operatorname{ptrA}$ was amplified with primers ptrA_forII/ptrA_revII from plasmid pSK275 (Szewczyk and Krappmann, 2010). For assembly of the gnoA deletion construct, a 3-fragment PCR according to the procedure described previously (Scharf etal., 2011) was performed with primers gnoA_5for and gnoA_3rev. For generating the $f h p B$ (AFUA_8G06080) deletion mutant the flanking regions were amplified with the primers fhpB_5for/fhpB_hph_5rev and fhpB_3rev/fhpB_hph_3for. Here, hygromycin resistance was used as selection marker and the hygromycin resistance cassette was amplified from plasmid pUChph (Liebmann et al., 2004) with the primer pair hph_for/hph_rev. Assembly of the $f h p B$ deletion construct was achieved by 3 -fragment PCR with primers fhpB_5for/fhpB_3rev. The deletion construct to create the $\Delta f h p A$ mutant was generated by amplifying the flanking regions of 
fhpA using the primer pairs fhpA_1(XmaI)/fhpA_2(Sfi ) and fhpA_3(SfiI)/fhpA_4(XhoI). The resulting DNA fragments were separately cloned into pCR2.1TOPO (Invitrogen, Germany) and subsequently isolated by restriction with XmaI/SfiI and SfiI/XhoI. The pyrithiamine resistance cassette was obtained by SfiI-restriction of plasmid pSK275. The fhpA DNA flanking fragments and the SfiI-digested ptrA gene were cloned into plasmid p123 (Spellig et al., 1996) restricted with XmaI/XhoI. The resulting plasmid was digested with $X m a I$ and $X h o I$ and the deletion cassette was used to transform $A$. fumigatus CEA17 $\Delta a k u B$ or $\Delta f h p B$ to create the $\triangle f h p A$ mutant and the $\triangle f h p A / \triangle f h p B$ double mutant, respectively. For creating the double mutants $\triangle f h p A / \triangle g n o A$ and $\triangle f h p B / \triangle g n o A$ the respective gnoA deletion cassettes conferring pyrithiamine or hygromycin resistance were employed to transform the mutants $\triangle f h p A$ and $\triangle f h p B$, respectively. The gnoA deletion cassette containing the hygromycin resistance cassette was generated by 3 -fragment PCR as described above employing primers gnoA_5for, gnoA_hph_5rev, gnoA_3rev, and gnoA_hph_3for. For complementation of the $\Delta g n o A$ mutant and construction of a GnoA-eGFP strain, the gnoA gene including $1 \mathrm{~kb}$ promoter sequence, was amplified from genomic A. fumigatus DNA using primers gnoA_Acc65I_for and gnoA_XmaI_rev. The DNA fragment was inserted in vector pJet1.2/blunt (Fermentas, Germany). The gnoA gene was inserted as Acc65I and XmaI fragment in plasmid pUCGH resulting in pUCGH_gnoA-egfp that was used to transform $A$. fumigatus $\triangle g n o A$. Complementation of the $\triangle f h p A$ mutant by construction of an FhpA-eGFP strain is described in Kroll et al. (2014). For generation of strain FhpB-eGFP containing the $f h p B-e g f p$ gene fusion under control of the constitutive otef promoter, the $f h p B$ gene was amplified by PCR using primers FhpB_XmaI_rev and FhpB_XmaI_for_otef. The DNA fragment was ligated via XmaI into plasmid pTH1 (a derivative of pUCGH carrying the pyrithiamine resistance cassette instead of the hygromycin resistance cassette) resulting in plasmid $\mathrm{pTH}$-fhpB-egfp that was used for transformation of A. fumigatus CEA10. All PCR reactions were performed using Phusion high-fidelity DNA polymerase (Finnzymes, Finland). All oligonucleotides used in this study are listed in Table S2.

\section{INHIBITION ZONE ASSAY}

Petri dishes with $10 \mathrm{ml}$ bottom-agar were covered with a layer of $10 \mathrm{ml}$ top-agar containing $1 \times 10^{8}$ conidia of the respective strain. In the center, $100 \mu \mathrm{l}$ of $45 \mathrm{mM}$ DETA-NO (Sigma, Germany) were filled in a hole of $10 \mathrm{~mm}$ diameter.

\section{GERMINATION ASSAY}

$1 \times 10^{4}$ conidia of $A$. fumigatus were cultivated in $50 \mu \mathrm{AMM}$ on cover slips and incubated at $37^{\circ} \mathrm{C}$ in a wet chamber. The ratio of germinated to non-germinated conidia was determined by light microscopy by counting 100 conidia/germlings per time point and strain. The assay was repeated three times.

\section{FLUORESCENCE MICROSCOPY}

Fifty microliter AMM were inoculated with $1 \times 10^{3}$ conidia of strain FhpA-eGFP or GnoA-eGFP on glass cover slips and incubated at $37^{\circ} \mathrm{C}$. To analyze dormant conidia, conidia were inoculated in $50 \mu \mathrm{L} \mathrm{H} \mathrm{H}_{2} \mathrm{O}$. For staining mitochondria, conidia of strain FhpB-eGFP were cultivated in 48 well plates with cover slips for $16 \mathrm{~h}$ in AMM. Fresh AMM with $500 \mathrm{nM}$ Mitotracker Deep Red (Invitrogen) was added and staining was done for $30 \mathrm{~min}$ at $37^{\circ} \mathrm{C}$. Before fluorescence microscopy, the samples were washed three times with PBS. Samples were analyzed using a Leica DMI 4000B and a DM 4500B microscope (Leica Microsystems, Germany). Images were taken with a Leica DFC480 or a DFC 340FX camera and analyzed by Leica LAS V.3.7 software.

\section{KILLING ASSAY}

Alveolar mouse macrophages (MH-S, ATCC CRL-2019) were grown in 12-well plates at a concentration of $7.5 \times 10^{5}$ cells per well over night at $37^{\circ} \mathrm{C}$ and $5 \%$ (v/v) $\mathrm{CO}_{2}$ in RPMI 1640 supplemented with $10 \%(\mathrm{v} / \mathrm{v}) \mathrm{FCS}$ and $50 \mu \mathrm{g} \mathrm{ml}^{-1}$ gentamycin (PAA Laboratories, Germany). After washing with PBS the cells were co-incubated with $1.5 \times 10^{6} \mathrm{~A}$. fumigatus conidia for $20 \mathrm{~min}$ at $4^{\circ} \mathrm{C}$ and then incubated for $6 \mathrm{~h}$ at $37^{\circ} \mathrm{C}$ at $5 \% \mathrm{CO}_{2}$. The samples were centrifuged with 5,000 $\mathrm{g}$ for $5 \mathrm{~min}$ and resuspended in $1 \mathrm{ml}$ ice-cold water and incubated for $10 \mathrm{~min}$ on ice. The samples were mixed and diluted 1:1000 with PBS/0.01\% (v/v) Tween 20. $100 \mu \mathrm{l}$ of the sample were plated on Sabouraud agar plates and colonies were counted after incubation for $24 \mathrm{~h}$ at $37^{\circ} \mathrm{C}$. For determination of CFU, five technical replicates were analyzed.

\section{MOUSE INFECTION MODEL}

Virulence of A. fumigatus mutant strains was tested in an established murine model for invasive pulmonary aspergillosis (Kupfahl et al., 2006; Schobel et al., 2010). Female CD-1 mice (Charles River, Germany) were immunosuppressed with cortisone acetate ( $25 \mathrm{mg}$ per mouse intraperitoneally; Sigma-Aldrich, Germany) 3 days before and on the day of infection. Mice were anesthetized and intranasally infected with $20 \mu \mathrm{l}$ of a fresh suspension containing $2 \times 10^{5}$ conidia. A control group was mock-infected with PBS. To induce neutropenia in mice, cyclophosphamide $(140 \mathrm{mg}$ $\mathrm{kg}^{-1}$; Sigma-Aldrich, Germany) was injected intraperitoneally on days $-4,-1,2,5,8$, and 11 , with an additional subcutaneous dose of cortisone acetate $\left(200 \mathrm{mg} \mathrm{kg}^{-1}\right)$ on day -1 . Mice were anesthetized and $3 \times 10^{4}$ conidia in $20 \mu \mathrm{l}$ PBS were applied intranasally.

The health status was monitored at least twice a day for 14 days and moribund animals, defined by severe dyspnoea, severe lethargy, or weight loss $>20 \%$, were sacrificed. Infections were performed with a group of 10 mice for each tested strain. Lungs from sacrificed animals were removed, and either stored in RNAlater (Qiagen, Germany) for RNA extraction or fixed in formalin and paraffin-embedded for histopathological analyses according to standard protocols. RNA isolation and first-strand cDNA synthesis from infected lungs was performed as described previously (Schmaler-Ripcke et al., 2009).

\section{Galleria mellonella INFECTION MODEL}

Virulence of the A. fumigatus $\Delta f h p A / \Delta f h p B$ mutant was tested in a non vertebrate infection model using fresh larvae of the greater wax moth Galleria mellonella (Kavanagh and Reeves, 2004). 
For each strain 30 larvae (Quality Bugs, Linnich, Germany) were infected with $5 \mu \mathrm{l}$ of an A. fumigatus spore suspension $\left(5 \times 10^{7}\right.$ spores $/ \mathrm{ml}$ in DPBS $+0.01 \%(\mathrm{v} / \mathrm{v})$ Tween 20$)$. Injection site was the lower right pseudopod. Larvae were checked for survival twice a day and accounted dead if nudging with forceps did not result in movement.

\section{STATISTICS}

The Student's $t$-test was used for significance testing of two groups. All significant differences are labeled with an asterisk $\left({ }^{*} p \leq 0.05\right.$; $* * p \leq 0.01)$.

\section{RESULTS \\ MOLECULAR CHARACTERIZATION OF THE A. fumigatus RNI DETOXIFICATION SYSTEM}

To elucidate the role of RNI in the pathogenicity of A. fumigatus, genes encoding putative RNI-detoxifying enzymes were identified. BLAST analyses revealed that the A. fumigatus genome contains two putative orthologs to the yeast flavohemoglobin YHB1, named FhpA (AFUA_4G03410) and FhpB (AFUA_8G06080). The FhpA and FhpB amino acid sequence revealed 40 and 36\% identity, respectively, to YHB1. FhpA and FhpB share $48 \%$ identical amino acids. As a third protein potentially involved in NO detoxification, GnoA (AFUA_2G01040) was identified as ortholog (73\% identity) of the nitrosoglutathione reductase Gno1 previously characterized in C. neoformans (Zhou et al., 2009).

Single and double deletion mutants of FhpA, FhpB, and GnoA encoding genes were generated (supplemental figures S1S7). To test the sensitivity of A. fumigatus against nitrosative stress, an inhibition zone assay using DETA-NO as NO donor was performed (Figure 2A). Strains $\triangle f h p A$ and $\triangle g n o A$ showed increased sensitivity toward DETA-NO compared to the wild type or corresponding complemented strains $f h p A-e g f p$ and gnoAegfp. Deletion of both flavohemoglobin-encoding genes $f h p A$ and $f h p B$ further increased RNI sensitivity. When grown on AMM agar plates with $\mathrm{NaNO}_{3}$ or $\mathrm{NaNO}_{2}$ as nitrogen source, all the mutants lacking the gnoA gene revealed a slight growth defect (data not shown), most likely due to the delay in germination of the $\triangle g n o A$ mutant (Figure 2B). The delay in germination was reduced when ammonium tartrate or glutamine were used as sole nitrogen source. Determination of the dry weight revealed that growth of all mutants was reduced in comparison to the wild type when grown in liquid medium with nitrate as sole nitrogen source. Replacing nitrate with glutamine complemented the growth defect in all flavohemoglobin mutants, except in the $\Delta g n o A$ mutant strain (data not shown). These results were supported by northern blot analyses monitoring mRNA steady state transcript levels of $f h p A$ and gnoA (Figure 3A). In AMM with nitrate as sole nitrogen source, the $f h p A$ gene was strongly expressed, indicating the need to detoxify RNI accumulating during nitrate metabolism. When the fungus was cultivated with ammonium tartrate or glutamine, transcription of $f h p A$ was comparatively low. In contrast to $f h p A$, transcript levels of $g n o A$ were similar in all media tested and were not affected by the nitrogen source. Transcripts of $f h p B$ were not detected under these conditions (data not shown).

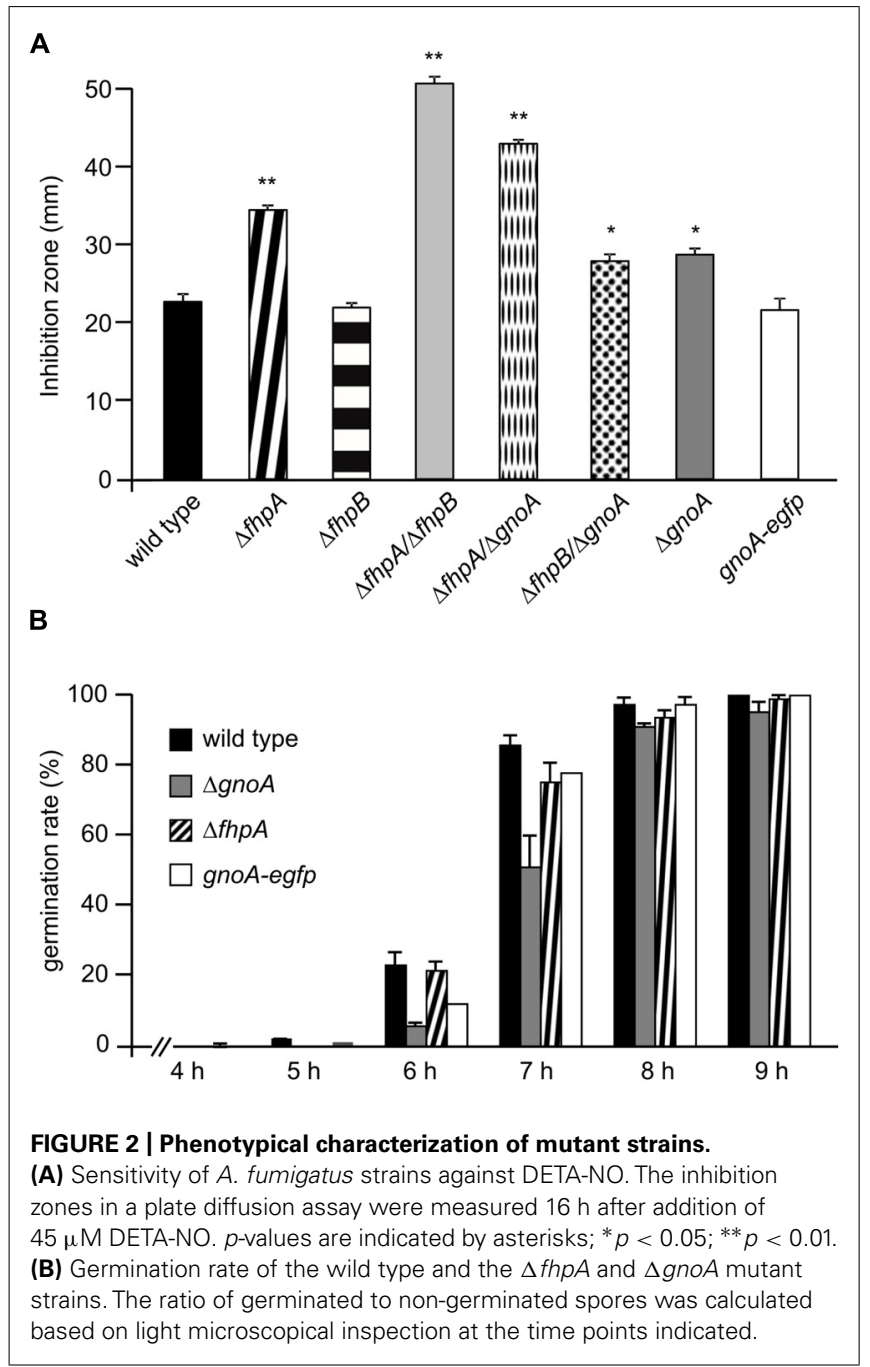

To proof whether genes encoding RNI detoxifying enzymes are involved in the infection process, their transcription was analyzed in lungs of infected mice. For this purpose, total RNA extracted from lungs of infected mice was isolated and transcription of genes was analyzed by RT-PCR (Figure 3B). As positive control, transcription of the A. fumigatus act1 gene (actin; AFUA_6G04740) was monitored. The genes $f h p A$ and gnoA were transcribed during the infection process, mRNA of $f h p B$ was not detected.

\section{LOCALIZATION OF FhpA, FhpB, and GnoA}

To determine the localization of FhpA and GnoA in A. fumigatus, the respective genes were fused to the egfp gene under control of their native promoter. Transformation of the $\triangle f h p A$ or the $\Delta g n o A$ strain with the $f h p A$-egfp or gnoA-egfp construct, respectively, resulted in transformants displaying wild-type phenotype, as tested by resistance to DETA-NO in agar plate diffusion assays (Figures S7 and S8). This data indicated that the respective fusion proteins were functional. Fluorescence microscopy revealed localization of both the FhpA-eGFP (Figure 4A) and the GnoA-eGFP protein (Figure 4B) in the cytoplasm of all A. fumigatus morphotypes, from conidia to hyphae. Due to 


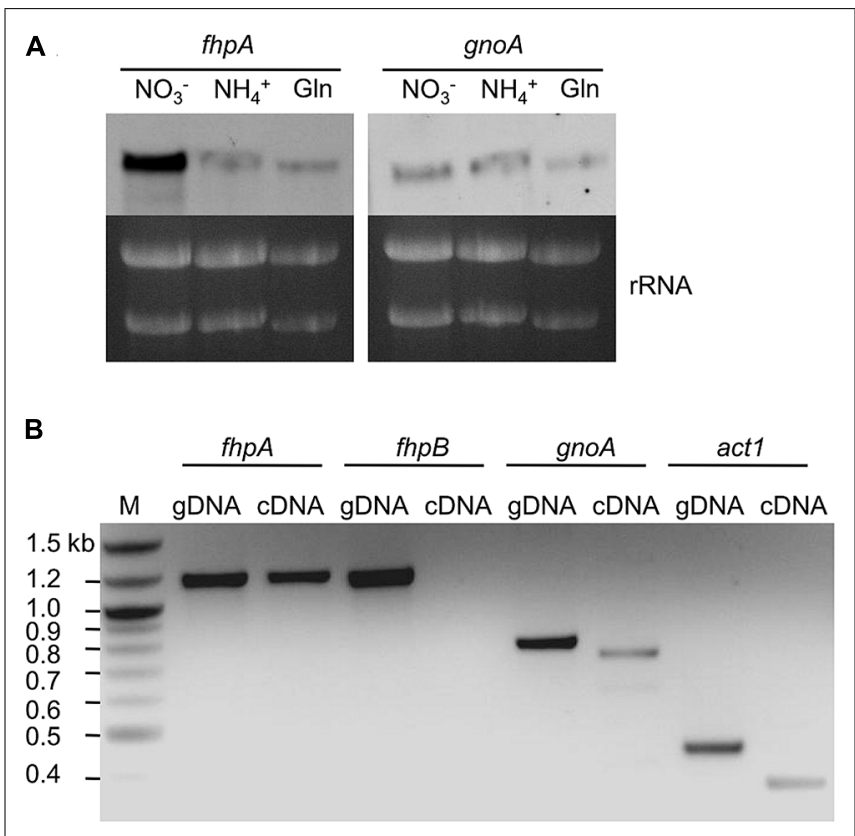

FIGURE 3 | Transcription of genes involved in RNI detoxification. (A) Northern blot analysis of fhpA and gnoA mRNA steady state levels in A. fumigatus cultivated for $16 \mathrm{~h}$ in $\mathrm{AMM}$ with different nitrogen sources. GIn, glutamine; rRNA was used as loading control. (B) PCR on cDNA of lungs of mice infected with $A$. fumigatus. Immunosuppressed mice were infected with $A$. fumigatus wild-type conidia and sacrificed at day 5 post infection. Genomic DNA (gDNA) of $A$. fumigatus was used as control template and transcription of the gene encoding $A$. fumigatus actin (act1) was used as positive control.

low transcriptional activity of the $f h p B$ promoter, fluorescence of a strain producing FhpB-eGFP was not detectable (data not shown). Therefore, a mutant was generated that produces FhpB-eGFP under control of the constitutive otef promoter (Figure S9). Fluorescence microscopy of strain FhpB-eGFP verified localization of this flavohemoglobin in the mitochondria (Figure 4C).

\section{CONFRONTATION OF MUTANT STRAINS WITH MACROPHAGES}

To analyze whether the ability to detoxify macrophage-derived RNI influences fungal survival, the killing rate of wild type and mutant conidia confronted with macrophages was determined. No significant differences in killing of $\triangle f h p A, \Delta f h p B$, or $\Delta g n o A$ conidia by cells of the macrophage cell line $\mathrm{MH}-\mathrm{S}$ were observed when compared to the wild type (Figure S10). This was also true for all double mutant strains deficient for RNI detoxification (data not shown).

\section{VIRULENCE OF RNI DETOXIFICATION MUTANTS}

The gnoA and $f h p A$ mutant strains were tested in a mouse infection model for pulmonary aspergillosis. In this model, mice were immunosuppressed solely with cortisone acetate, i.e., neutrophils, that are able to produce $\mathrm{NO}$, are still recruited to the site of infection. Survival of infected animals was monitored over a period of 14 days (Figure 5A). No significant differences in survival of mice infected with the mutant strains compared to mice infected with the wild type and the corresponding complemented strains occurred. Furthermore, histopathology of lungs of infected mice did not reveal any differences with regard to fungal growth or the recruitment of neutrophils (data not shown). In an alternative infection model, neutropenic mice, treated with cyclophosphamide, were infected with conidia of the $\triangle f h p A / \triangle g n o A$ double deletion strain. Again, no difference in survival was detected compared to mice infected with wildtype conidia (Figure 5B). Additionally, virulence of the strain $\triangle f h p A / \triangle f h p B$ was analyzed in a non-vertebrate model, using larvae of the greater wax moth $G$. mellonella. Also in this model, there was no difference in killing of larvae by the wild type or by the RNI detoxification mutants (Figure 5C).

\section{DISCUSSION}

Immune cells eliminate pathogens by different mechanisms including the release of ROI during the oxidative burst and the production of RNI (Bogdan, 2011). The role of these reactive intermediates in defense against an infection with A. fumigatus is still a matter of debate. In a previous study that reported the impact of ROI and RNI on killing of A. fumigatus by alveolar macrophages, the authors speculated that ROI, but not RNI, are important in fungal killing (Philippe et al., 2003). Furthermore, triple deletion mutants of fungal superoxide dismutates showed a higher sensitivity against menadion and an impaired killing by alveolar macrophages (Lambou et al., 2010). However, more recent studies clearly showed that ROI are not directly involved in killing of $A$. fumigatus as ROI detoxification mutants of $A$. fumigatus are still fully virulent in mouse infection models (Lamarre et al., 2007; Lessing et al., 2007).

In C. neoformans two RNI detoxification systems exist (de Jesús-Berríos etal., 2003). The first system is represented by flavohemoglobins, which convert NO to nitrate via a nitrosyl intermediate. Similarly, as shown here, A. fumigatus harbors two flavohemoglobins, namely FhpA and FhpB. The resulting nitrate is metabolized by nitrate reductase $\mathrm{NiaD}$ to nitrite and then to ammonium via nitrite reductase NiiA (see Figure 1). The second system for RNI detoxification uses $S$-nitrosoglutathione (GSNO) reductases that reduce GSNO to ammonium and glutathione disulphide (GSSG). Here, GnoA was found to be involved in fungal germination. Testing the effect of different $\mathrm{NO}$ donors revealed that NO is able to negatively influence germination (Kunert, 1995). Therefore, the delayed germination in the mutants analyzed here, can be directly assigned to accumulation of RNI during initiation of germination. Consistently, all mutants with the exception of $\Delta f h p B$, were more sensitive against $\mathrm{NO}$ when challenged with DETA-NO.

Screening fungal genome databases revealed that filamentous fungi like Neurospora crassa, Gibberalla zeae and also several Aspergillus sp. carry two putative genes encoding flavohemoglobins (te Biesebeke et al., 2010). In A. fumigatus, one of these genes, $f h p A$, seems to play a major role because it can compensate the lack of $f h p B$. This is in concordance with the results obtained from $A$. nidulans where mutants for $f h p A$ but not $f h p B$ grown on minimal medium with nitrite as sole nitrogen source showed drastically reduced growth (Schinko et al., 2010). Furthermore, similar to FhpA, the nitrate/nitrite reductase is localized in the 


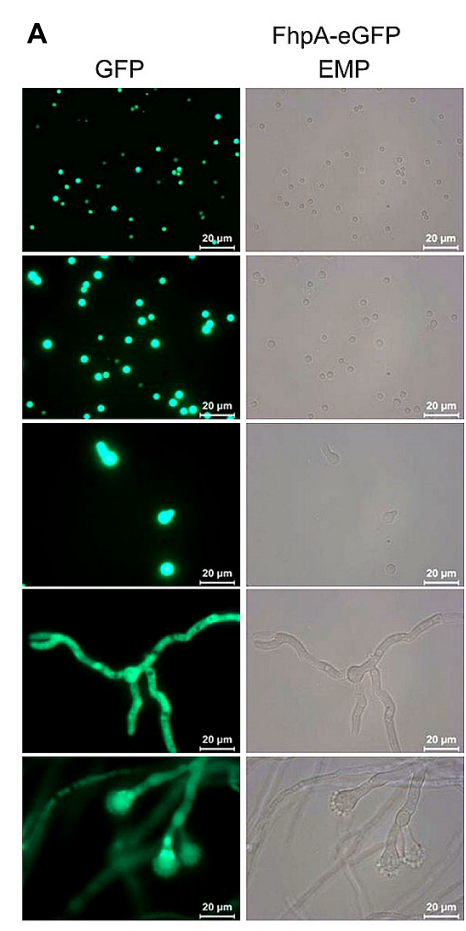

C

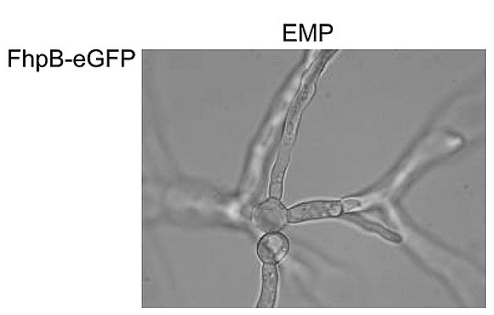

Overlay

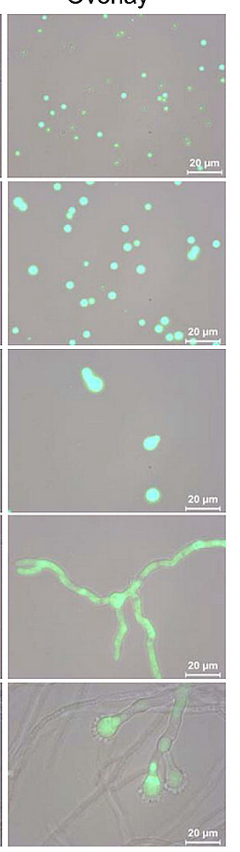

B
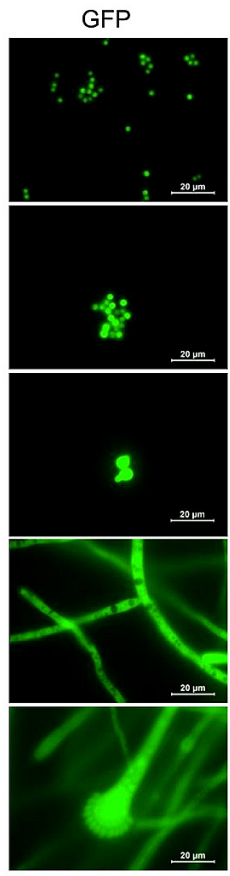

GFP
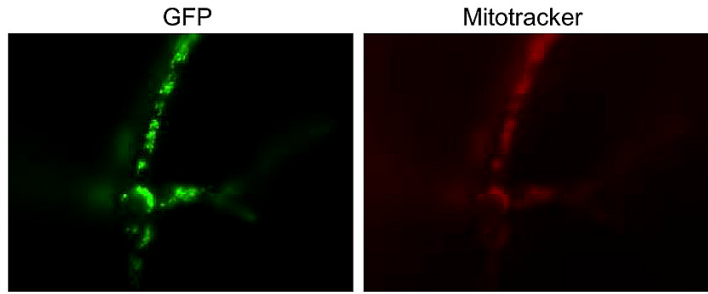

FIGURE 4 | Localization of FhpA-eGFP, GnoA-eGFP, and FhpB-eGFP using fluorescence microscopy. (A) Fluorescence of strains FhpA-eGFP and (B) GnoA-eGFP was monitored in resting conidia (stored in $\mathrm{H}_{2} \mathrm{O}$ ), in swollen conidia (incubation for $3 \mathrm{~h}$ in $\mathrm{AMM}$ ), germlings (7 h AMM), hyphae (16 h AMM), and conidiophores (24 h AMM). (C) Fluorescence microscopy and staining with Mitotracker Red revealed localization of FhpB-eGFP in the mitochondria. cytoplasm, further supporting a predominant role of FhpA in RNI detoxification, whereas FhpB was localized in mitochondria. Consistently, in $A$. oryzae deletion of the $f h p B$ homolog $f h p 2$ did not affect NO sensitivity. Transcript levels of $f h p 2$ were extremely low and in accordance with this observation fluorescence of an Fhp2-eGFP fusion protein was only detectable when an inducible promoter was employed. Fhp2 is located in the mitochondria and is putatively involved in detoxification of NO derived from nitrite metabolism (Zhou et al., 2011). In A. fumigatus, FhpB was recently found to be part of the proteome of resting conidia, indicating a function of this flavohemoglobin in the initial phase of stress response toward RNI (Teutschbein etal., 2010). This might also provide an explanation for the synergistic NO sensitivity of the $f h p A / f h p B$ double mutant.

The function of flavohemoglobins seems to be specific for detoxification of RNI because neither $\Delta f h p A$ nor $\Delta f h p B$ showed altered sensitivity against oxidative stress-inducing agents like menadione, diamide, or $\mathrm{H}_{2} \mathrm{O}_{2}$ (data not shown). A similar result was found for the flavohemoglobin YHB1 of $S$. cerevisiae, because an $S c \Delta y h b 1$ mutant also exhibited specific activity restricted to RNI detoxification (Lewinska and Bartosz, 2006).

Similar to A. nidulans, A. fumigatus RNI detoxification mutants exhibited a growth defect when cultivated in AMM with nitrate as nitrogen source, which was rescued when the fungus was grown with other nitrogen sources like glutamine or ammonium tartrate. Metabolism of different nitrogen sources resulted in generation of different levels of RNI (Schinko et al., 2010), which was also reflected by different $f h p A$ and gnoA mRNA steady state levels depending on the nitrogen source.

A first hint for a potential role of NO-detoxifying enzymes in virulence of $A$. fumigatus was obtained by detection of $f h p A$ and gnoA transcripts in lungs of infected mice. Furthermore, Sugui etal. (2008) showed upregulation of gnoA-transcription in conidia confronted with neutrophils. In general, ROI, and RNI levels in the tissue increase during infection (Evans et al., 1993). RNI fulfill important functions in the defense against 


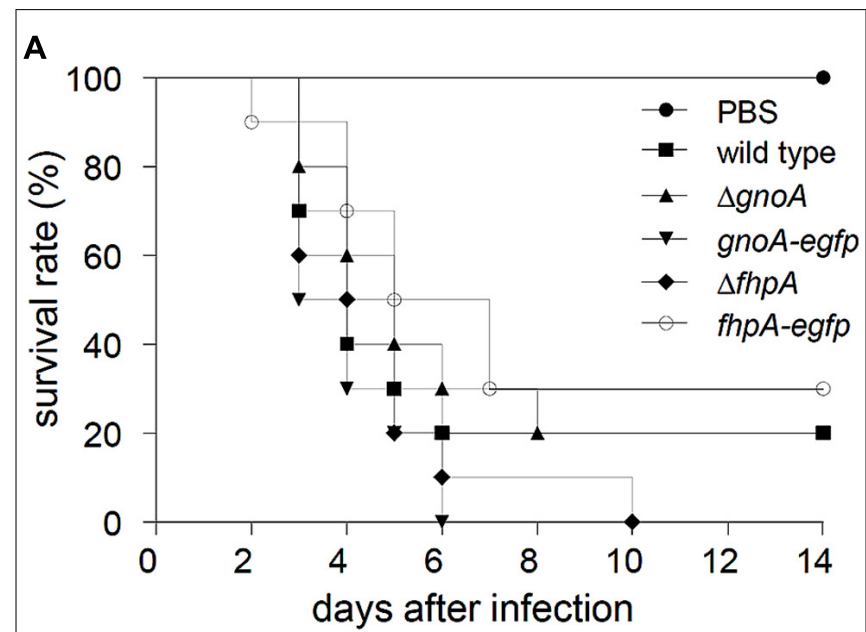

B

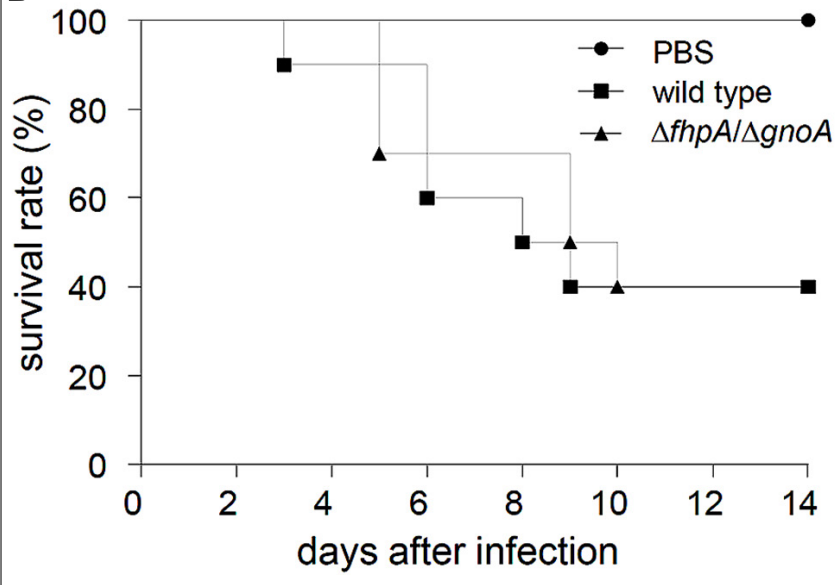

C

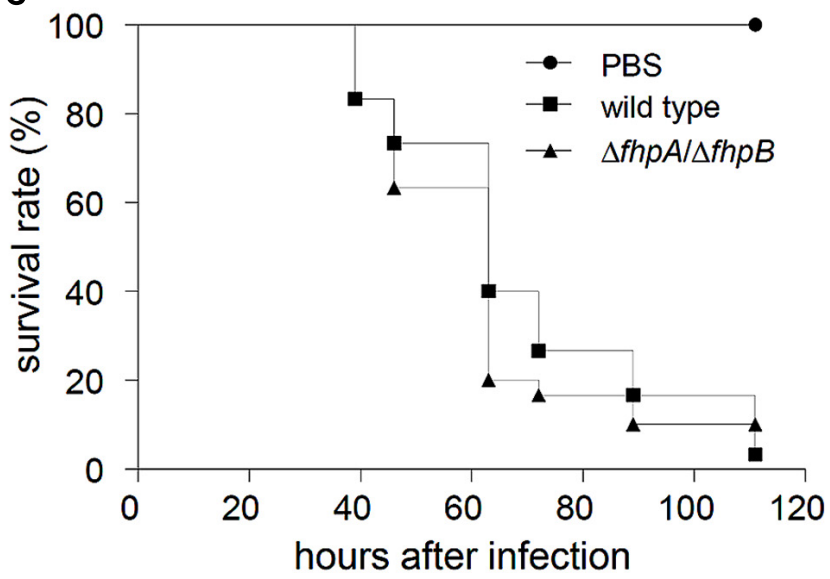

FIGURE 5 | Virulence studies. (A) Cortisone acetate treated mice were infected with $2 \times 10^{5}$ conidia of the indicated $A$. fumigatus strains. As control, a group of mice $(n=5)$ remained uninfected (inhalation of PBS). Survival was monitored for 14 days. (B) Neutropenic mice were infected with $3 \times 10^{4}$ conidia of the indicated $A$. fumigatus strains. (C) Fresh larvae of the greater wax moth $G$. mellonella were infected with $5 \times 10^{5}$ conidia of the wild type and the $\triangle f h p A / \triangle f h p B$ mutant, respectively. As mock control PBS was used. Survival of larvae was monitored for 5 days. microorganisms. This was shown for bacteria (Chakravortty and Hensel, 2003), protozoa (Souza etal., 2010; Yeo et al., 2010) and also for human pathogenic fungi (Atochina et al., 2004; Hromatka etal., 2005; Nittler et al., 2005; Missall et al., 2006; Gonzalez etal., 2007). Miramon etal. (2012) showed that in C. albicans the $y h b 1 \Delta$ mutant is more susceptible to killing by neutrophils and wild-type sensitivity could be regained by addition of the NO scavenger carboxy-PTIO. Gross et al. (1999) reported that A. fumigatus enhanced the production of RNI in alveolar macrophages. However, as shown here, in co-incubation assays with macrophages of the MH-S cell line no differences in survival between NO detoxification-deficient mutants conidia and the wild-type conidia were detected. Certainly, the possibility remains that the level of RNI sensitivity in the mutant strains is not sufficient to exclude any impact of RNI detoxification on the interaction with immune cells. At least in the infection models applied in our study, deletion of genes involved in RNI detoxification did not alter virulence of $A$. fumigatus. To study $A$. fumigatus virulence in mice, two different models for immunosuppression are currently used. In the leukopenic model, animals are immunosuppressed by reiterate application of cyclophosphamide and a single low-dose of cortisone acetate, resulting in neutrophil depletion. In the second model immunosuppression is solely induced by administration of high doses of cortisone acetate, resulting in an impaired function of macrophages but still allowing recruitment of neutrophils. In our study, both murine infection models revealed wild-type pathogenicity of the RNI detoxification mutants. It may be the case that high levels of corticosteroid treatment impacts NO production capacity of immune cells. Therefore, in addition the G. mellonella infection model was employed. Haemocytes from G. mellonella are able to produce NO when they are confronted with pathogens or LPS (Krishnan et al., 2006; Semenova et al., 2014). Also in this invertebrate model that does not require immunosuppression, virulence of the mutants was not affected. Taken together, the A. fumigatus genome encodes two flavohemoglobins, FhpA and FhpB, and the $S$-nitrosoglutathione reductase GnoA, involved in RNI detoxification. FhpA and GnoA were found to represent the primarily responsible enzymes in A. fumigatus to counteract RNI stress. However, in different infection models RNI detoxification mutants revealed wild-type pathogenicity, implying that the ability to detoxify host-derived RNI is at least no major prerequisite for virulence of $A$. fumigatus.

\section{ACKNOWLEDGMENTS}

Carmen Schult and Silke Steinbach are acknowledged for excellent technical assistance. The work was funded by the excellence graduate school Jena School for Microbial Communication (JSMC; www.jsmc.uni-jena.de), the International Leibniz Research School for Microbial and Biomolecular Interactions (ILRS; www.ilrs.hki-jena.de), and the CRC/Transregio 124 FungiNet (www.funginet.de).

\section{SUPPLEMENTARY MATERIAL}

The Supplementary Material for this article can be found online at: http://www.frontiersin.org/journal/10.3389/fmicb.2014.00469/ abstract 


\section{REFERENCES}

Atochina, E. N., Gow, A. J., Beck, J. M., Haczku, A., Inch, A., Kadire, H., et al. (2004). Delayed clearance of Pneumocystis carinii infection, increased inflammation, and altered nitric oxide metabolism in lungs of surfactant protein-D knockout mice. J. Infect. Dis. 189, 1528-1539. doi: 10.1086/383130

Bergmann, S., Funk, A. N., Scherlach, K., Schroeckh, V., Shelest, E., Horn, U., et al. (2010). Activation of a silent fungal polyketide biosynthesis pathway through regulatory cross talk with a cryptic nonribosomal peptide synthetase gene cluster. Appl. Environ. Microbiol. 76, 8143-8149. doi: 10.1128/AEM.-00683-10

Bogdan, C. (2011). "Reactive oxygen and reactive nitrogen intermediates in the immune system," in Immunology of Infectious Diseases, eds S. H. Kaufmann, B. Rouse, and D. Sacks (Washington, DC: ASM Press), 69-84.

Bogdan, C., Rollinghoff, M., and Diefenbach, A. (2000). Reactive oxygen and reactive nitrogen intermediates in innate and specific immunity. Curr. Opin. Immunol. 12, 64-76. doi: 10.1016/S0952-7915(99)00052-7

Brakhage, A. A. (2005). Systemic fungal infections caused by Aspergillus species: epidemiology, infection process and virulence determinants. Curr. Drug Targets 6, 875-886. doi: 10.2174/138945005774912717

Brakhage, A. A., Bruns, S., Thywissen, A., Zipfel, P. F., and Behnsen, J. (2010). Interaction of phagocytes with filamentous fungi. Curr. Opin. Microbiol. 13, 409-415. doi: 10.1016/j.mib.2010.04.009

Brakhage, A. A., and Van den Brulle, J. (1995). Use of reporter genes to identify recessive trans-acting mutations specifically involved in the regulation of Aspergillus nidulans penicillin biosynthesis genes. J. Bacteriol. 177, 2781-2788.

Brown, A. J., Haynes, K., and Quinn, J. (2009). Nitrosative and oxidative stress responses in fungal pathogenicity. Curr. Opin. Microbiol. 12, 384-391. doi: 10.1016/j.mib.2009.06.007

Chakravortty, D., and Hensel, M. (2003). Inducible nitric oxide synthase and control of intracellular bacterial pathogens. Microbes Infect. 5, 621-627. doi: 10.1016/S1286-4579(03)00096-0

Chiranand, W., McLeod, I., Zhou, H., Lynn, J. J., Vega, L. A., Myers, H., et al. (2008). CTA4 transcription factor mediates induction of nitrosative stress response in Candida albicans. Eukaryot. Cell 7, 268-278. doi: 10.1128/EC.00240-07

da Silva Ferreira, M. E., Kress, M. R., Savoldi, M., Goldman, M. H., Härtl, A., Heinekamp, T., et al. (2006). The akuBKU80 mutant deficient for nonhomologous end joining is a powerful tool for analyzing pathogenicity in Aspergillus fumigatus. Eukaryot. Cell 5, 207-211. doi: 10.1128/EC.5.1.207-211.2006

de Jesús-Berríos, M., Liu, L., Nussbaum, J. C., Cox, G. M., Stamler, J. S., and Heitman, J. (2003). Enzymes that counteract nitrosative stress promote fungal virulence. Curr. Biol. 13, 1963-1968. doi: 10.1016/j.cub.2003.10.029

Evans, T. G., Thai, L., Granger, D. L., and Hibbs, J. B. (1993). Effect of in vivo inhibition of nitric oxide production in murine leishmaniasis. J. Immunol. 151, 907-915.

Fang, F. C. (1997). Perspectives series: host/pathogen interactions. Mechanisms of nitric oxide-related antimicrobial activity. J. Clin. Invest. 99, 2818-2825. doi: 10.1172/JCI119473

Fernández, M. R., Biosca, J. A., and Parés, X. (2003). S-nitrosoglutathione reductase activity of human and yeast glutathione-dependent formaldehyde dehydrogenase and its nuclear and cytoplasmic localisation. Cell. Mol. Life Sci. 60, 1013-1018. doi: 10.1007/s00018-003-3025-x

Gonzalez, A., Restrepo, A., and Cano, L. E. (2007). Role of iron in the nitric oxidemediated fungicidal mechanism of IFN- $\gamma$-activated murine macrophages against Paracoccidioides brasiliensis conidia. Rev. Inst. Med. Trop. Sao Paulo 49, 11-16. doi: 10.1590/S0036-46652007000100003

Gross, N. T., Nessa, K., Camner, P., and Jarstrand, C. (1999). Production of nitric oxide by rat alveolar macrophages stimulated by Cryptococcus neoformans or Aspergillus fumigatus. Med. Mycol. 37, 151-157. doi: 10.1080/j.1365280X.1999.00209.x

Hromatka, B. S., Noble, S. M., and Johnson, A. D. (2005). Transcriptional response of Candida albicans to nitric oxide and the role of the YHB1 Gene in nitrosative stress and virulence. Mol. Biol. Cell 16, 4814-4826. doi: 10.1091/mbc.E05-05-0435

Kavanagh, K., and Reeves, E. P. (2004). Exploiting the potential of insects for in vivo pathogenicity testing of microbial pathogens. FEMS Microbiol. Rev. 28, 101-112. doi: 10.1016/j.femsre.2003.09.002

Krishnan, N., Hyrsl, P., and Simek, V. (2006). Nitric oxide production by hemocytes of larva and pharate prepupa of Galleria mellonella in response to bacterial lipopolysaccharide: cytoprotective or cytotoxic? Comp. Biochem. Physiol. C Toxicol. Pharmacol. 142, 103-110. doi: 10.1016/j.cbpc.2005.10.016
Kroll, K., Pahtz, V., Hillmann, F., Vaknin, Y., Schmidt-Heck, W., Roth, M., et al. (2014). Identification of hypoxia-inducible target genes of Aspergillus fumigatus by transcriptome analysis reveals cellular respiration as important contributor to hypoxic survival. Eukaryot. Cell doi: 10.1128/EC.00084-14 [Epub ahead of print].

Kunert, J. (1995). Effect of nitric oxide donors on survival of conidia, germination and growth of Aspergillus fumigatus in vitro. Folia Microbiol. (Praha) 40, 238-244. doi: 10.1007/BF02814199

Kupfahl, C., Heinekamp, T., Geginat, G., Ruppert, T., Hartl, A., Hof, H., et al. (2006). Deletion of the gliP gene of Aspergillus fumigatus results in loss of gliotoxin production but has no effect on virulence of the fungus in a low-dose mouse infection model. Mol. Microbiol. 62, 292-302. doi: 10.1111/j.1365-2958.2006. 05373.x

Lamarre, C., Ibrahim-Granet, O., Du, C., Calderone, R., and Latgé, J.-P. (2007). Characterization of the SKN7 ortholog of Aspergillus fumigatus. Fungal Genet. Biol. 44, 682-690. doi: 10.1016/j.fgb.2007.01.009

Lambou, K., Lamarre, C., Beau, R., Dufour, N., and Latge, J. P. (2010). Functional analysis of the superoxide dismutase family in Aspergillus fumigatus. Mol. Microbiol. 75, 910-923. doi: 10.1111/j.1365-2958.2009.07024.x

Lessing, F., Kniemeyer, O., Wozniok, I., Loeffler, J., Kurzai, O., Haertl, A., et al. (2007). The Aspergillus fumigatus transcriptional regulator AfYapl represents the major regulator for defense against reactive oxygen intermediates but is dispensable for pathogenicity in an intranasal mouse infection model. Eukaryot. Cell 6, 22902302. doi: 10.1128/EC.00267-07

Lewinska, A., and Bartosz, G. (2006). Yeast flavohemoglobin protects against nitrosative stress and controls ferric reductase activity. Redox Rep. 11, 231-239. doi: 10.1179/135100006X154987

Liebmann, B., Müller, M., Braun, A., and Brakhage, A. A. (2004). The cyclic AMPdependent protein kinase A network regulates development and virulence in Aspergillus fumigatus. Infect. Immun. 72, 5193-5203. doi: 10.1128/IAI.72.9.51935203.2004

Miramon, P., Dunker, C., Windecker, H., Bohovych, I. M., Brown, A. J., Kurzai, O., et al. (2012). Cellular responses of Candida albicans to phagocytosis and the extracellular activities of neutrophils are critical to counteract carbohydrate starvation, oxidative and nitrosative stress. PLOS ONE 7:e52850. doi: 10.1371/journal.pone.0052850

Missall, T. A., Pusateri, M. E., Donlin, M. J., Chambers, K. T., Corbett, J. A., and Lodge, J. K. (2006). Posttranslational, translational, and transcriptional responses to nitric oxide stress in Cryptococcus neoformans: implications for virulence. Eukaryot. Cell 5, 518-529. doi: 10.1128/EC.5.3.518-529.2006

Nittler, M. P., Hocking-Murray, D. Foo, C. K., and Sil, A. (2005). Identification of Histoplasma capsulatum transcripts induced in response to reactive nitrogen species. Mol. Biol. Cell 16, 4792-4813. doi: 10.1091/mbc.E05-05-0434

Philippe, B., Ibrahim-Granet, O., Prévost, M. C., Gougerot-Pocidalo, M. A., Sanchez Perez, M., Van der Meeren, A., et al. (2003). Killing of Aspergillus fumigatus by alveolar macrophages is mediated by reactive oxidant intermediates. Infect. Immun. 71, 3034-3042. doi: 10.1128/IAI.71.6.3034-3042.2003

Scharf, D. H., Remme, N., Habel, A., Chankhamjon, P., Scherlach, K., Heinekamp, T., et al. (2011). A dedicated glutathione S-transferase mediates carbon-sulfur bond formation in gliotoxin biosynthesis. J. Am. Chem. Soc. 133, 12322-12325. doi: $10.1021 /$ ja201311d

Schinko, T., Berger, H., Lee, W., Gallmetzer, A., Pirker, K., Pachlinger, R., et al. (2010). Transcriptome analysis of nitrate assimilation in Aspergillus nidulans reveals connections to nitric oxide metabolism. Mol. Microbiol. 78, 720-738. doi: 10.1111/j.1365-2958.2010.07363.x

Schmaler-Ripcke, J., Sugareva, V., Gebhardt, P., Winkler, R., Kniemeyer, O., Heinekamp, T., et al. (2009). Production of pyomelanin, a second type of melanin, via the tyrosine degradation pathway in Aspergillus fumigatus. Appl. Environ. Microbiol. 75, 493-503. doi: 10.1128/AEM.02077-08

Schobel, F., Jacobsen, I. D., and Brock, M. (2010). Evaluation of lysine biosynthesis as an antifungal drug target: biochemical characterization of Aspergillus fumigatus homocitrate synthase and virulence studies. Eukaryot. Cell 9, 878-893. doi: 10.1128/EC.00020-10

Semenova, A. D., Glazachev, Y. I., Slepneva, I. A., and Glupov, V. V. (2014). Quantitative determination of nitric oxide production in haemocytes: nitrite reduction activity as a potential pathway of NO formation in haemolymph of Galleria mellonella larvae. Nitric Oxide 37, 46-52. doi: 10.1016/j.niox.2013.12.011

Souza, A., Giudice, A., Pereira, J., Guimaraes, L., de Jesus, A., de Moura, T., et al. (2010). Resistance of Leishmania (Viannia) braziliensis to nitric oxide: correlation 
with antimony therapy and TNF-alpha production. BMC Infect. Dis. 10:209. doi: 10.1186/1471-2334-10-209

Spellig, T., Bottin, A., and Kahmann, R. (1996). Green fluorescent protein (GFP) as a new vital marker in the phytopathogenic fungus Ustilago maydis. Mol. Gen. Genet. 252, 503-509. doi: 10.1007/BF02172396

Sugui, J. A., Kim, H. S., Zarember, K. A., Chang, Y. C., Gallin, J. I., Nierman W. C., et al. (2008). Genes differentially expressed in conidia and hyphae of Aspergillus fumigatus upon exposure to human neutrophils. PLoS ONE. 3:e2655. doi: 10.1371/journal.pone.0002655

Szewczyk, E., and Krappmann, S. (2010). Conserved regulators of mating are essential for Aspergillus fumigatus cleistothecium formation. Eukaryot. Cell 9, 774-783. doi: 10.1128/EC.00375-09

te Biesebeke, R., Levasseur, A., Boussier, A., Record, E., van den Hondel, C. A., and Punt, P. J. (2010). Phylogeny of fungal hemoglobins and expression analysis of the Aspergillus oryzae flavohemoglobin gene fhbA during hyphal growth. Fungal Biol. 114, 135-143. doi: 10.1016/j.mycres.2009.08.007

Tekaia, F., and Latgé, J.-P. (2005). Aspergillus fumigatus: saprophyte or pathogen? Curr. Opin. Microbiol. 8, 385-392. doi: 10.1016/j.mib.2005.06.017

Teutschbein, J., Albrecht, D., Potsch, M., Guthke, R., Aimanianda, V., Clavaud, C., et al. (2010). Proteome profiling and functional classification of intracellular proteins from conidia of the human-pathogenic mold Aspergillus fumigatus. J. Proteome Res. 9, 3427-3442. doi: 10.1021/pr9010684

Wartenberg, D., Lapp, K., Jacobsen, I. D., Dahse, H. M., Kniemeyer, O., Heinekamp, T., et al. (2011). Secretome analysis of Aspergillus fumigatus reveals Asp-hemolysin as a major secreted protein. Int. J. Med. Microbiol. 301, 602-611. doi: 10.1016/j.ijmm.2011.04.016

Weidner, G., d'Enfert, C., Koch, A., Mol, P. C., and Brakhage, A. A. (1998). Development of a homologous transformation system for the human pathogenic fungus Aspergillus fumigatus based on the pyrG gene encoding orotidine 5'-monophosphate decarboxylase. Curr. Genet. 33, 378-385. doi: $10.1007 / \mathrm{s} 002940050350$
Yeo, T. W., Lampah, D. A., Tjitra, E., Gitawati, R., Darcy, C. J., Jones, C., et al. (2010). Increased asymmetric dimethylarginine in severe falciparum malaria: association with impaired nitric oxide bioavailability and fatal outcome. PLoS Pathog. 6:e1000868. doi: 10.1371/journal.ppat.1000868

Zhou, S., Fushinobu, S., Kim, S. W., Nakanishi, Y., Maruyama, J., Kitamoto, K., etal. (2011). Functional analysis and subcellular location of two flavohemoglobins from Aspergillus oryzae. Fungal Genet. Biol. 48, 200-207. doi: 10.1016/j.fgb.2010.08.011

Zhou, S., Fushinobu, S., Nakanishi, Y., Kim, S. W., Wakagi, T., and Shoun, H. (2009). Cloning and characterization of two flavohemoglobins from Aspergillus oryzae. Biochem. Biophys. Res. Commun. 381, 7-11. doi: 10.1016/j.bbrc.2009.01.112

Conflict of Interest Statement: The authors declare that the research was conducted in the absence of any commercial or financial relationships that could be construed as a potential conflict of interest.

Received: 26 June 2014; accepted: 18 August 2014; published online: 11 September 2014.

Citation: Lapp K, Vödisch M, Kroll K, Strassburger M, Kniemeyer O, Heinekamp T and Brakhage AA (2014) Characterization of the Aspergillus fumigatus detoxification systems for reactive nitrogen intermediates and their impact on virulence. Front. Microbiol. 5:469. doi: 10.3389/fmicb.2014.00469

This article was submitted to Fungi and Their Interactions, a section of the journal Frontiers in Microbiology.

Copyright (C) 2014 Lapp, Vödisch, Kroll, Strassburger, Kniemeyer, Heinekamp and Brakhage. This is an open-access article distributed under the terms of the Creative Commons Attribution License (CC BY). The use, distribution or reproduction in other forums is permitted, provided the original author(s) or licensor are credited and that the original publication in this journal is cited, in accordance with accepted academic practice. No use, distribution or reproduction is permitted which does not comply with these terms. 